\title{
The Inaugural Issue
}

\author{
E. Ray Dorsey \\ Center for Health and Technology, University of Rochester Medical Center, Rochester, NY, USA
}

The inaugural issue of Digital Biomarkers discusses how mobile technologies are reshaping measurement of neurodegenerative conditions, introduces the Network of Digital Evidence in Health (NODE), and highlights perspectives from multiple disciplines and industries that the journal seeks to represent. The first issue has multiple pieces highlighting how digital biomarkers are increasingly providing insights into neurodegenerative disorders. Such applications are important for at least three reasons. First, neurodegenerative disorders are increasingly common and are now the leading source of disability globally. Second, unlike many other conditions, central nervous system disorders lack good functional (e.g., echocardiography), plasma (e.g., CD4 counts), and in many cases (e.g., Parkinson disease) imaging biomarkers. Third, the brain and spinal cord are difficult to access and while increasing in value, cerebrospinal fluid markers currently have limited utility in tracking disease progression. Consequently, subjective, insensitive, episodic rating scales dominate evaluations of many neurodegenerative disorders, including Alzheimer disease, amyotrophic lateral sclerosis, and Huntington disease.

To help address these limitations, Giggins and colleagues conducted a review of physical activity monitors for individuals with neurological disorders. Heldman and colleagues investigated a FDA-cleared device for evaluation of Parkinson disease in the home. In addition, Adams and colleagues evaluated wearable accelerometers for individuals with Parkinson disease, Huntington disease, and prodromal Huntington disease in the clinic and at home. While Adams and colleagues did not find any differences in those with prodromal Huntington disease and controls in a small pilot study, their evaluation of prodromal states with digital biomarkers is likely to be replicated in a wide range of clinical disorders, including Alzheimer disease, diabetes, and hypertension. This work lays the foundation for possible identification of individuals with these prodromal states and evaluation of interventions aimed at preventing the onset of disease.

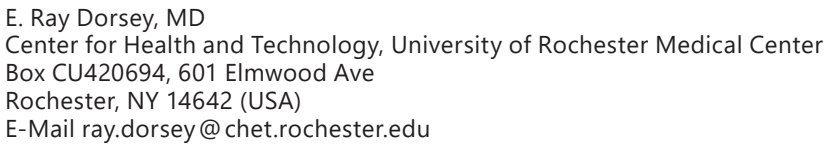


Also in this issue, Makhni, Atreja, and colleagues introduce NODE. This important effort addresses an important limitation in the field - evidence that demonstrates the value of mHealth and digital biomarkers to improve health. We are fortunate that Dr. Ashish Atreja, the Chief Technology Innovation and Engagement Officer at the Icahn School of Medicine at Mount Sinai, will serve as a section editor of Digital Biomarkers to enable sharing of digital biomarker studies across academia and industry, facilitate adoption of these tools, and support rigorous and fast evaluation of mobile technologies.

Finally, this issue features contributions from many disciplines including computer science, engineering, medicine, and physics and multiple industries, including academia, pharmaceutical, and technology. As a result of this diversity, we have perspectives on how to ensure patient engagement, use these tools in clinical trials, and how the digital divide affects the field. In addition to academic papers, this issue features interviews with Dr. Robert M. Califf, the former Commissioner of the U.S. Food and Drug Administration and long-time proponent of health computing, and Dr. William J. Marks, Jr., the head of clinical neurology at Verily Life Sciences, a company that, as a colleague recently remarked, may over the next decade become the largest health care company in the world. Digital Biomarkers seeks to foster this diverse set of communities and voices as we all work to use these new digital tools to improve the health. We look forward to hearing yours. 